
\title{
28 Research Suare \\ Upregulation of TIPE2 attenuates macrophage activation and neuroinflammation after intracerebral hemorrhage in mice
}

\section{Shudong Liu}

Universitatsklinikum Munster Klinik fur Neurologie mit Institut fur Translationale Neurologie

Jie Wang

Universitatsklinikum Munster Klinik fur Neurologie mit Institut fur Translationale Neurologie

\section{Wenyan Li}

Universitatsklinikum Munster Klinik fur Neurologie mit Institut fur Translationale Neurologie

Hui Shi

Universitatsklinikum Munster Klinik fur Neurologie mit Institut fur Translationale Neurologie

Changlong Zhou

Universitatsklinikum Munster Klinik fur Neurologie mit Institut fur Translationale Neurologie

Ge Tang

neurology

Jiangwei Zhang

Institut National de Neurologie Mongi-Ben Hamida

zhao yang ( $\square$ yangzhao5140@sohu.com )

Universitatsklinikum Zentrum fur Neurologie

\section{Research}

Keywords: TIPE2, macrophage, neuroinflammation, ICH

Posted Date: August 10th, 2020

DOI: https://doi.org/10.21203/rs.3.rs-54788/v1

License: (c) (1) This work is licensed under a Creative Commons Attribution 4.0 International License. Read Full License 


\section{Abstract}

Background: Intracerebral hemorrhage $(\mathrm{ICH})$ is a serious disease with high mortality and morbidity, and effective treatment is limited. A large amount of evidence suggests that the inflammatory response contributes to secondary brain damage following ICH. TIPE2 is an essential negative regulator of both innate and adaptive immunity, and depletion of TIPE2 causes inflammatory disease. However, the possible role of TIPE2 following ICH has not been reported.

Methods: In this study, we investigated TIPE2 levels and inflammation in macrophages treated with erythrocyte lysate in vitro, and we observed proinflammatory cytokine production, BBB disruption, cerebral water content and neurological damage in $\mathrm{ICH}$ mice in vivo.

Results: We found that TIPE2 levels were significantly decreased in erythrocyte lysate-treated macrophages compared to control macrophages. Upregulation of TIPE2 decreased macrophage activation and cytokine production and accelerated brain damage in ICH mice. In addition, upregulation of TIPE2 attenuated proinflammatory cytokine production, BBB disruption, and severe brain inflammation after $\mathrm{ICH}$.

Conclusion: The results demonstrated that TIPE2 was negatively correlated with the pathogenesis of ICH, which prevented brain injury and attenuated deleterious inflammatory responses following ICH. TIPE2 might serve as a novel target for $\mathrm{ICH}$ therapy.

\section{Introduction}

Intracerebral hemorrhage $(\mathrm{ICH})$ is a severe neurological disease with high morbidity and mortality $[1,2,3]$. $\mathrm{ICH}$ often induces severe neurological impairment because of inflammation-mediated secondary brain damage $[4,5,6]$. Brain inflammation following $\mathrm{ICH}$ is characterized by the accumulation of activated inflammatory cells. These activated cells produce inflammatory mediators, including chemokines, cytokines, proteases, and other proinflammatory molecules [7, 8, 9]. Among the factors that contribute to brain inflammation, macrophage activation plays an essential role in the secondary damage after ICH $[10,11,12]$.

TIPE2 (tumor necrosis factor (TNF)-induced protein 8-like 2) plays an important role in maintaining immune homeostasis by negatively regulating $T$ cell receptor and Toll-like receptor (TLR) signaling [13, $14,15]$. TIPE2 is preferentially expressed in lymphoid tissues, macrophages and lymphocytes. TIPE2 has been identified to be involved in inflammatory and infectious diseases, such as hepatitis $B$, systemic lupus erythematosus, and asthma $[16,17,18]$.

However, whether TIPE2 plays a role in the regulation of inflammation following ICH has not been reported. Considering TIPE2 is essential for the maintenance of immune homeostasis, we hypothesize that TIPE2 might contribute to inflammation following ICH. In this study, we explore TIPE2 levels and the related effects on inflammation following $\mathrm{ICH}$. 


\section{Materials And Methods}

Experimental animals

C57BL/6 mice were generated and kept in our laboratory according to a previous study. All mice used were male and 8-10 weeks old and weighed $22-25 \mathrm{~g}$. The mice were maintained in individual cages under controlled conditions $\left(22 \pm 2{ }^{\circ} \mathrm{C} ; 55 \%\right.$ humidity) with free access to food and fresh water. All animal experiments were approved by the Ethics Committee of Chongqing Medical University.

Primary cell cultures

Cortical neuronal cultures were prepared from the whole cerebral cortices of C57BL/6 mouse embryos (E16). After removal of the meninges, the tissue was digested with $0.005 \%$ trypsin/0.002\% EDTA (10 min, $37^{\circ} \mathrm{C}$ ), mechanically dissociated, and centrifuged at $1000 \times \mathrm{g}$ for $5 \mathrm{~min}$. The cell pellet was resuspended in neurobasal medium (Gibco) containing B27 serum-free supplement (Gibco) and $500 \mathrm{~N} \mathrm{~m} \mathrm{~L} \mathrm{-glutamine.}$ A total of $2 \times 10^{5}$ cells/well were seeded on sterile poly-L-lysine (Sigma-Aldrich)-coated glass cover slips in a 24-well plate and incubated at $37{ }^{\circ} \mathrm{C}$ and $5 \% \mathrm{CO}_{2}$. After $1 \mathrm{~h}$, the culture medium was changed completely. The purity of the neuronal cultures was $>95 \%$, as confirmed by random staining with neuronal and glial markers. Five days after plating, the neurons had developed a dense network of extensions. To isolate primary macrophage cells, the cerebral hemispheres of 1-day-old postnatal mice were digested with $0.1 \%$ trypsin. The cells were seeded into a six-well plate coated with poly-L-lysine and cultured with Dulbecco's modified Eagle's medium (DMEM; Sigma, St. Louis, MO, USA) containing 10\% fetal bovine serum (FBS; HyClone, Logan, UT, USA). The culture medium was refreshed twice per week for 2 weeks. Macrophages were detached by gentle shaking and filtered through a nylon mesh to remove astrocytes. After centrifugation at $1000 \times \mathrm{g}$ for $10 \mathrm{~min}$, the cells were resuspended in fresh DMEM supplemented with $10 \%$ FBS and plated at a final density of $5 \times 10^{5}$ cells $/ \mathrm{mL}$ on a poly-L-lysine coated 6well culture plate. The following day, the cells were subjected to experiments. The cell purity was determined by immunohistochemical staining using a macrophage-specific CD11b antibody. The macrophage cultures used were $>95 \%$ pure.

Lentivirus encoding TIPE2 construction

A TIPE2 expressing lentivirus gene transfer vector was constructed by Genechem Co., Ltd., Shanghai, China. The identity of the vector was confirmed by PCR and sequencing analysis. The recombinant TIPE2 expressing lentivirus (LV-TIPE2) and the control lentivirus (Lv-CON) were prepared at a titer of $10^{9} \mathrm{TU}$ (transfection unit)/ml.

Lentivirus transduction and detection assay

Macrophage were transduced with lentiviral vectorsat a multiplicity

of infection (MOI) of 50 in complete medium containing polybrene 
$(5 \mu \mathrm{g} / \mathrm{ml})$ at $37^{\circ} \mathrm{C}$ and $5 \% \mathrm{CO}_{2}$ for $18-24 \mathrm{~h}$. The cells were then

washed and cultured in fresh medium containing 10\% FBS. Cells

were harvested 3 days following transduction.

Preparation of erythrocyte lysates

The spleens were removed from C57BL/ 6 mice. Single-cell suspensions of splenocytes were prepared using stainless steel mesh screens. Then, $1 \times 10^{5}$ splenocytes were incubated with $1 \mathrm{ml}$ of red blood cell lysis solution for $20 \mathrm{~min}$ and centrifuged at $2000 \mathrm{rpm}$ for $10 \mathrm{~min}$. The supernatants were utilized as erythrocyte lysates.

Cell treatment

Macrophages $\left(1 \times 10^{5}\right)$ were stimulated with $10 \mu \mathrm{L}$ of erythrocyte lysate. After 3 days, the supernatants were removed and further analyzed for cytokine production by ELISA. Neurons were cultured in a 96-well plate at a density of $1 \times 10^{4}$ cells per well. For the toxicity experiments, neurons were serum-starved for $4 \mathrm{~h}$ and then treated with a mixture of macrophage-conditioned media. For the MTT and apoptosis assays, the cells were treated for $48 \mathrm{~h}$.

$\mathrm{ICH}$ model

Briefly, mice were anesthetized by an intraperitoneal injection of $400 \mathrm{mg} / \mathrm{kg}$ chloral hydrate and fixed on a

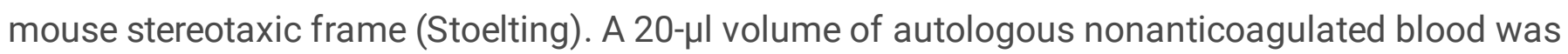
collected from the tail vein of the mice and then injected into the caudate nucleus at $0.8 \mathrm{~mm}$ anterior to bregma, $2 \mathrm{~mm}$ lateral to bregma the left, and $3.5 \mathrm{~mm}$ deep under stereotactic guidance at a rate of $2 \mu \mathrm{l} / \mathrm{min}$ over a period of $10 \mathrm{~min}$. The needle was held in place for $10 \mathrm{~min}$ after injection, and the microsyringe was pulled out after the blood had coagulated. The craniotomy was then sealed with bone wax, and the scalp was closed with sutures. Body temperature was maintained at $37^{\circ} \mathrm{C}$ throughout the procedure, and the mice were given free access to food and water after they woke up. The mice that died due to anesthesia were excluded.

Administration of TIPE2 Lentivirus

To study the effects of TIPE2, mice received an intracerebral ventricular injection of TIPE2 Lentivirus or control lentivirus ( $1 \times 10^{9}$ plaque forming units [Pfu], $2 \mu \mathrm{l}$ of a $10 \mathrm{mg} / \mathrm{ml}$ solution prepared in $\left.0.9 \% \mathrm{NaCl}\right)$ 10 min after $\mathrm{ICH}$ and sacrificed $48 \mathrm{~h}$ after $\mathrm{ICH}$.

\section{RT-qPCR}

Total RNA was extracted from mouse brain tissue by using TRIzol (Invitrogen, Carlsbad, CA, USA) according to the manufacturer's instructions. Reverse transcription was performed with a First Strand 
cDNA Synthesis kit (Fermentas, Vilnius, Lithuania). Real-time PCR was performed on an ABI 7300 Sequence Detection System with a SYBR-Green PCR kit (both Applied Biosystems; Thermo Fisher Scientific, Inc.). All primers used in this study were purchased from Sangon Biotech Co., Ltd. (Shanghai, China), and the sequences were as follows: TIPE2, forward 5'-TGA AAC TCA GGT CCG CTT CT-3' and reverse 5'-TCC TAG TGC TGC CTC CAA CT-3'. For qPCR, $1 \mu$ of CDNA, 1 nM primers and $12.5 \mu$ of $2 \times$ SYBR Green were mixed to obtain a final volume of $25 \mu$ l. Thermal cycling was performed as follows: 40 cycles at $95^{\circ} \mathrm{C}$ for $30 \mathrm{sec}, 56^{\circ} \mathrm{C}$ for $30 \mathrm{sec}$ and $72^{\circ} \mathrm{C}$ for $30 \mathrm{sec}$. Relative gene expression levels were determined using the comparative $\mathrm{Cq}$ method with GAPDH as the control.

Western blot analysis

Total protein was isolated from ipsilateral lesional brain tissues using ice-cold RIPA buffer. The protein concentrations were measured with the BCA Protein Assay Kit (Forevergen Biosciences). The protein samples were separated using sodium dodecylsulfate polyacrylamide gel electrophoresis (SDS-PAGE) and transferred to polyvinylidene difluoride membranes. The proteins were detected by incubation with primary antibodies followed by horseradish peroxidase-conjugated secondary antibodies. The immunoblots were visualized using ECL plus Western Blotting Detection Reagents (Pluslight, Forevergen Biosciences, China). GAPDH (1:1000, Cell Signaling Technology, USA) was employed as the loading control. Densitometry analysis was performed with the use of ImageJ software with normalization to GAPDH.

Enzyme-linked immunosorbent assay (ELISA)

Macrophages $\left(1 \times 10^{5}\right)$ were stimulated with $10 \mu \mathrm{L}$ of erythrocyte lysate. After 3 days, the supernatants were removed and further analyzed for cytokine production by ELISA. ELISA was performed according to the manufacturer's instructions (Dakewe Biotech, Shenzhen, China) to assess the concentrations of TNFa, IL-1 $\beta$ and IL- 6 in the culture supernatant.

MTT assay

The viability of neurons was assessed using a 3-(4,5-dimethyl-2 -thiazolyl) - 2,5-diphenyl-2H-tetrazolium bromide (MTT, Sigma-Aldrich) assay. After $48 \mathrm{~h}$, MTT reagent was added to the wells, and the cells were incubated for $4 \mathrm{~h}$ at $37^{\circ} \mathrm{C}$ and $5 \% \mathrm{CO}_{2}$. After centrifugation, the supernatant was removed from each well. The colored formazan crystal produced from MTT was dissolved with $0.15 \mathrm{~mL}$ of DMSO, then the A490 optical density (OD) value was measured with a multiscanner autoreader (Dynatech MR 5000; Dynatech Laboratories, Chantilly, VA, USA). The absorbance was measured at $570 \mathrm{~nm}$. The mean of the readings of triplicate wells was taken as the value. The OD value for the control cultures was considered $100 \%$ viability, and the viability of the other samples is expressed as a percentage of the viability of the control cultures.

Flow cytometry analysis of apoptosis 
An annexinV-fluorescein isothiocyanate (FITC) apoptosis detection kit (OncogeneResearch Products, Boston, MA) was used to detect apoptosis. The cells were seeded in 1-mL flasks and incubated in DMEM supplemented with $10 \%$ fetal bovine serum until they reached approximately $90 \%$ confluence. Then, the cells were harvested, washed with ice-cold PBS twice, and resuspended in binding buffer (10 mM Hepes, $\mathrm{pH}$ 7.4, $50 \mathrm{mM} \mathrm{NaCl}, 2.5 \mathrm{mM} \mathrm{CaCl}_{2}, 1 \mathrm{mM} \mathrm{MgCl}_{2}$, and $4 \%$ bovine serum albumin). Annexin V-fluorescein isothiocyanate $(0.5 \mathrm{mg} / \mathrm{mL})$ and propidium iodide $(0.6 \mathrm{mg} / \mathrm{mL})$ were then added to a $250 \mathrm{~mL}$ aliquot $(1 \times$ $10^{6}$ cells) of this cell suspension according to the manufacturer's protocol. After a 15 -minute incubation in the dark at room temperature, the stained cells were immediately analyzed with a flow cytometer (Beckman Coulter, USA). All of the samples were assayed in triplicate, and the cell apoptosis rate was calculated using the following formula: apoptosis rate $=$ (apoptotic cell number $/$ total cell number) $\times$ $100 \%$.

\section{Caspase assay}

To detect caspase-3-like protease activities, the ApoAlert Caspase-3 Colorimetric Assay kit (Clontech, Palo Alto, USA) was used. Cytosolic lysates were prepared $48 \mathrm{~h}$ following transfection and incubated with $50 \mathrm{~mm}$ p-nitroanilide (pNA) conjugated to the caspase cleavage site Asp-Glu-Val-Asp (DEVD) for $1 \mathrm{~h}$ at $37^{\circ} \mathrm{C}$. Hydrolyzed pNA was detected using a Multiscan MS colorimeter (Thermo Labsystems, Vantaa, Finland) at $405 \mathrm{~nm}$. For control experiments, the lysates were incubated with $10 \mathrm{mM}$ caspase-3 inhibitor DEVD-fmk (Clontech) for 30 min before addition of the substrate.

Histochemical evaluation of macrophage activation

Three days after $\mathrm{ICH}$, the animals were deeply anesthetized with pentobarbital and transcardially perfused with $0.9 \%$ saline followed by $4 \%$ paraformaldehyde in $0.1 \mathrm{M}$ phosphate buffer (PBS, pH 7.4). After the mice were perfused and fixed, the perihematomal regions of the cerebral tissues were collected, fixed in $4 \%$ paraformaldehyde for $24 \mathrm{~h}$, dehydrated in $30 \%$ sucrose solution for $48 \mathrm{~h}$, embedded, frozen, and cut into $25-\mu \mathrm{m}$ sections using a Leica CM1900 cryostat. The perihematomal sections were treated with $3 \% \mathrm{H}_{2} \mathrm{O}_{2}$ in $0.01 \mathrm{M}$ phosphate-buffered saline (PBS) and preincubated in $5 \%$ normal goat serum. The samples were then incubated in a primary antibody solution containing a rat anti-lba antibody (Serotec, Fullerton, CA, USA, 1:200) overnight at $4{ }^{\circ} \mathrm{C}$. After washing, the samples were incubated in a secondary IgG antibody (1:200) for $1 \mathrm{~h}$ at room temperature (RT). Finally, the sections were incubated in horseradish peroxidase (HRP)-streptavidin (1:200) for $1 \mathrm{~h}$ at $\mathrm{RT}$, and the color reaction was conventionally developed with diaminobenzidine (DAB) and $\mathrm{H}_{2} \mathrm{O}_{2}$. Six representative sections from the brain of each animal were selected. IPP6.0 image processing software (Media Cybernetics, MD, USA) was utilized to count the number of positive cells.

\section{Evans Blue Extravasation}

Briefly, under anesthesia, Evans blue dye ( $2 \%, 5 \mathrm{~mL} / \mathrm{kg}$; Aladdin, Shanghai, China) was injected into the left femoral vein over a period of $>2$ minutes and allowed to circulate for 60 minutes. Then, the mice were euthanized by intracardial perfusion of sterile saline. The brain samples were weighed, homogenized in 
sterile saline, and centrifuged at $15000 \times \mathrm{g}$ for 30 minutes. After that, an equal volume of trichloroacetic acid was added to the resultant supernatant. The samples were then incubated overnight at $4{ }^{\circ} \mathrm{C}$ and centrifuged again at $15000 \times \mathrm{g}$ for 30 minutes. The resultant supernatant was spectrophotometrically quantified for extravasated Evans blue dye at $620 \mathrm{~nm}$.

Brain water content measurement

Brain water content was measured in mouse cerebral tissues after ICH. Briefly, mice were randomly sampled from each group and anesthetized by intraperitoneal injection of chloral hydrate $(n=5)$. Next, the cerebral tissues were removed, and the water on the surface of the cerebral tissues was blotted with filter paper. The brain samples were immediately weighed with an electric analytic balance to obtain the wet weight and then dried at $100{ }^{\circ} \mathrm{C}$ for $24 \mathrm{~h}$ to obtain the dry weight. Brain water content was calculated using the following formula: brain water content $(\%)=$ (wet weight - dry weight) $/$ wet weight $\times 100 \%$.

Evaluation of Neurological Scores

Neurological scores were determined by the Neurological Severity Scale, a composite of motor, sensory, reflex, and balance tests. Neurological function was graded on a scale of 1 to 18; a score of 1 point was awarded for the inability to perform a test or for the lack of a tested reflex. The higher the score, the more severe the injury (normal score 2-3; maximal deficit score 18).

\section{Statistical analysis}

All experiments were repeated three times. The significance of differences between groups was determined by two-tailed Student's $t$ test and two-way ANOVA. P values of less than 0.05 were considered statistically significant.

\section{Results}

\section{TIPE2 lentivirus improved TIPE2 levels following erythrocyte lysate treatment and $\mathrm{ICH}$.}

We analyzed TIPE2 levels in macrophages after erythrocyte lysate treatment by RT-qPCR and western blot analysis. The data demonstrated that TIPE2 levels in macrophages were significantly decreased after erythrocyte lysate treatment. However, TIPE2 lentivirus improved TIPE2 levels compared with control group after erythrocyte lysis treatment (Fig. 1A). Furthermore, we detected the alterations in TIPE2 in the mouse brain 3 ds following $\mathrm{ICH}$. The results demonstrated that TIPE2 levels in the mouse brain were significantly decreased after ICH. However, TIPE2 lentivirus improved TIPE2 levels compared with control group after ICH (Fig. 1B). 


\section{TIPE2 lentivirus decreased the macrophage inflammatory response in vitro}

To analyze the role of TIPE2 in macrophage inflammation, we utilized erythrocyte lysate to treat macrophages and detected cytokine expression by ELISA. We found that erythrocyte lysate treatment significantly increased TNF-a, IL-1 $\beta$, and IL- 6 levels in macrophages compared with PBS treatment. However, TIPE2 lentivirus decreased TNF-a, IL-1 $\beta$, and IL- 6 levels compared with control group after erythrocyte lysis treatment (Fig. 2). These data suggest that TIPE2 decreases the macrophage- mediated inflammatory response and TIPE2 lentivirus decreased the macrophage inflammatory response in vitro.

\section{TIPE2 lentivirus inhibited macrophage-mediated inflammatory injury in neurons}

To assess the effect of TIPE2 on macrophage-mediated inflammatory injury in neurons, we utilized the MTT assay to assess erythrocyte lysate-induced toxicity of macrophages to neurons. The data demonstrated that when neurons were treated with conditioned medium from erythrocyte lysate-treated macrophages, the cell viability significantly decreased. However, when the cells were treated with conditioned medium from TIPE2 lentivirus transduced macrophages, cell viability increased (Fig. 3A). We further detected neuron apoptosis and the specific apoptotic pathways. Neurons were treated with conditioned medium from erythrocyte lysate-treated macrophages for 2 days, and the cell apoptosis ratio and caspase activity were detected by flow cytometry and a colorimetric assay. The results demonstrated that the cell apoptosis rate and caspase-3 activity increased significantly in neurons treated with conditioned medium from erythrocyte lysate-treated macrophages compared with that from TIPE2 lentivirus transduced macrophages (Fig. 3B and C). Therefore, the results suggest that TIPE2 attenuates macrophage-mediated inflammatory injury in neurons in vitro.

\section{TIPE2 lentivirus attenuated macrophage accumulation in the perihematomal region}

Macrophage accumulation is involved in brain inflammatory damage. To detect the effect of TIPE2 in macrophage accumulation, we detected Iba-1-positive macrophages in the perihematomal brain tissue 3 days after $\mathrm{ICH}$. Our data indicated that the number of macrophages in the perihematomal brain tissues was increased after 3 days in ICH WT mice compared with sham controls. However, the number of macrophages in TIPE2 lentivirus transduced mice was decreased compared with that in WT mice after ICH (Fig. 4). These results suggest that TIPE2 attenuates macrophage accumulation in the perihematomal region in $\mathrm{ICH}$ mice. 


\section{TIPE2 lentivirus inhibited inflammation in the perihematomal region}

To identify the role of TIPE2 in inflammation following ICH, we analyzed the mRNA and protein levels of inflammatory factors, such as TNF-a, IL-1 $\beta$ and IL- 6 . The results demonstrated that the mRNA and protein levels of inflammatory factors in the perihematomal brain tissues were significantly increased in ICH WT mice compared with sham controls. However, the mRNA and protein levels of inflammatory factors in TIPE2 lentivirus transduced mice were decreased compared with those in WT mice after ICH (Fig. 5). These data indicate that TIPE2 inhibits inflammation in the perihematomal region.

\section{TIPE2 lentivirus attenuated neurological damage following $\mathrm{ICH}$}

To further explore the effect of TIPE2 on neurological damage following ICH, we analyzed BBB integrity, brain water content and the neurological deficit scores of ICH mice. The data indicated that BBB injury, brain water content and neurological deficit scores of ICH mice were much higher than sham controls. However, BBB injury, brain water content and neurological deficit scores in TIPE2 lentivirus transduced $\mathrm{ICH}$ mice were much lower than control group (Fig. 6). These results suggest that TIPE2 attenuates neurological damage following $\mathrm{ICH}$.

\section{Discussion}

In the current study, we present the following evidence, which has not been reported previously: (1) Erythrocyte lysate treatment and ICH decreased TIPE2 levels. (2) TIPE2 decreased the macrophagemediated inflammatory response in vitro. (3) TIPE2 inhibited macrophage-mediated inflammatory injury to neurons. (4) TIPE2 inhibited inflammation in the perihematomal region. (5) TIPE2 attenuated neurological damage following $\mathrm{ICH}$.

Hematoma following ICH can directly compress brain tissue, which further induces mechanical damage and secondary brain injury $[19,20,21]$. Inflammation contributes to brain damage following $\mathrm{ICH}$, leading to macrophage activation and blood-brain barrier (BBB) disruption [22, 23, 24]. Therefore, inhibiting inflammatory responses after $\mathrm{ICH}$ might be a promising approach for reducing secondary brain injury.

TIPE2 belongs to the TNFAIP8 family and has been proven to be overexpressed in mice with experimental autoimmune encephalomyelitis [25]. Many studies suggest that TIPE2 has the ability to negatively regulate the innate and adaptive immune responses $[26,27,28]$. TIPE2 is preferentially expressed in lymphoid tissues, and TIPE2-deficient cells are sensitive to Toll-like receptor (TLR) and T cell receptor (TCR) activation [29]. TLR4 is activated by many endogenous ligands, such as heme and fibrinogen, which are produced in the brain after ICH. Therefore, TIPE2 might be regulated by erythrocyte lysates. A previous study showed that TIPE2 negatively regulates inflammation by switching arginine metabolism 
from nitric oxide synthase to arginase in macrophages [30, 31, 32]. More evidence has suggested that TIPE2 might inhibit M1 macrophage differentiation but induce M2 macrophage polarization [33, 34]. However, the underlying mechanism by which TIPE2 inhibits macrophage inflammation following ICH remains unclear.

To explore the effect of ICH on macrophages, we first utilized erythrocyte lysate to treat macrophages and analyzed macrophage inflammatory responses. We found that erythrocyte lysate significantly increased cytokine levels in macrophages compared with WT macrophages. However, TIPE2 can inhibit the inflammatory response of macrophages induced by erythrocyte lysate. Furthermore, we found that TIPE2 inhibited macrophage-mediated inflammatory injury to neurons in vitro. These data suggest that TIPE2 might directly contribute to the immunosuppressive action of ICH-induced macrophage inflammation. Second, to investigate the role of TIPE2 in the macrophage accumulation and neuroinflammation following $\mathrm{ICH}$, we analyzed Iba-1-positive macrophages and inflammatory factors in the perihematomal brain tissues after ICH. These results suggested that TIPE2 attenuated macrophage accumulation and inflammatory factors in the perihematomal region after $\mathrm{ICH}$. In addition, we observed TIPE2 transduced ICH mice exhibited decreased macrophage accumulation. Finally, to detect the specific role of TIPE2 in ICH-induced brain damage, we assessed BBB integrity, brain water content and the neurological deficit scores of ICH mice. The results indicated that TIPE2 attenuated neurological damage following ICH.

Previous studies have shown that ICH can activate MAPK signaling through phosphorylation of ERK1/2, p38 and JNK proteins, leading to inflammatory cytokine production [35]. In addition, phosphorylation of ERK, p38, and JNK is increased in TIPE2-silenced macrophages stimulated with MP. Furthermore, MAPK inhibitors, including a JNK inhibitor, P38 inhibitor and ERK inhibitor, can partially attenuate the negative role of TIPE2 in MP-triggered cytokine production in THP-1 cells[36]. Therefore, we postulate that the downregulation of TIPE2 expression after ICH might contribute to the elevated levels of inflammatory cytokines, possibly via the APK signaling pathway. However, the relevant molecular mechanisms by which TIPE2 inhibits intracellular signaling pathways remain to be further investigated.

\section{Conclusion:}

The data indicated that TIPE2 contributes to the pathogenesis of $\mathrm{ICH}$, which prevents brain injury and attenuates inflammation following $\mathrm{ICH}$. In addition, TIPE2 might represent a promising target for ICH therapy.

\section{Abbreviations}

None

\section{Declarations}


The datasets used and analyzed in the current study are available from the corresponding author upon reasonable request.

\section{Contributions}

ZY conceptualized and designed the study. SDL, WYL and HS performed the experiments and analyzed data. GT designed and performed all cytokine analysis experiments. JWZ performed immunohistochemistry, cell quantification, and data analysis, and prepared the figures. All authors critically reviewed the manuscript, approved the final manuscript as submitted and agree to be accountable for all aspects of the work.

\section{Acknowledgements}

The authors gratefully acknowledge the technical assistance of Mr. Zhongyan Huang.

\section{Competing interest}

The authors declare that they have no competing interests.

\section{Funding}

This work was supported by the Yongchuan Natural Science Foundation (Ycstc, 2019nb02026). The funding supported the design of the study; the collection, analysis, and interpretation of the data; the writing of the manuscript.

\section{Ethics approval and consent to participate}

All animals received care in compliance with the Principles of Laboratory Animal Care and National Standards.

\section{Availability of data and materials}

Please contact author for data requests.

\section{Consent for publication}

Not applicable.

\section{Competing interests}

The authors declare that they have no competing interests.

\section{References}


1. van Ginneken V, Engel P, Fiebach JB, Audebert HJ, Nolte $\mathrm{CH}$, et al. Prior antiplatelet therapy is not associated with larger hematoma volume or hematoma growth in intracerebral hemorrhage. Neurol Sci. 2018;39:745-8.

2. Tamborska A, Poon MTC, Al-Shahi Salman R. Characteristics of Randomized Trials Focusing on Stroke due to Intracerebral Hemorrhage: Systematic Review. Stroke. 2018;49:594-600.

3. Kim DB, Park SK, Moon BH, Cho BR, Jang DK, et al. Comparison of craniotomy and decompressive craniectomy in large supratentorial intracerebral hemorrhage. J Clin Neurosci. 2018;50:208-13.

4. Zhang F, Zhang C. Rnf112 deletion protects brain against intracerebral hemorrhage (ICH) in mice by inhibiting TLR-4/NF-kappaB pathway. Biochem Biophys Res Commun. 2018;507:43-50.

5. Lan X, Han X, Liu X, Wang J. Inflammatory responses after intracerebral hemorrhage: From cellular function to therapeutic targets. J Cereb Blood Flow Metab. 2019;39:184-6.

6. Qiao HB, Li J, Lv LJ, Nie BJ, Lu P, et al. Eupatilin inhibits macrophage activation and attenuates brain injury in intracerebral hemorrhage. Exp Ther Med. 2018;16:4005-9.

7. Li X, Wang T, Zhang D, Li H, Shen H, et al. Andrographolide ameliorates intracerebral hemorrhage induced secondary brain injury by inhibiting neuroinflammation induction. Neuropharmacology. 2018;141:305-15.

8. Chen C, Zhou F, Zeng L, Jiang Z, Hu Z. Methylene blue offers neuroprotection after intracerebral hemorrhage in rats through the PI3K/Akt/GSK3beta signaling pathway. J Cell Physiol. 2019;234:5304-18.

9. Zhang Z, Wu Y, Yuan S, Zhang P, Zhang J, et al. Glutathione peroxidase 4 participates in secondary brain injury through mediating ferroptosis in a rat model of intracerebral hemorrhage. Brain Res. 2018;1701:112-25.

10. Bonsack Ft, Alleyne CH Jr, Sukumari-Ramesh S. Augmented expression of TSPO after intracerebral hemorrhage: a role in inflammation? J Neuroinflammation. 2016;13:151.

11. Yang J, Ding S, Huang W, Hu J, Huang S, et al. Interleukin-4 Ameliorates the Functional Recovery of Intracerebral Hemorrhage Through the Alternative Activation of Macrophage/Macrophage. Front Neurosci. 2016;10:61.

12. Chen M, Li X, Zhang X, He X, Lai L, et al. The inhibitory effect of mesenchymal stem cell on bloodbrain barrier disruption following intracerebral hemorrhage in rats: contribution of TSG-6. J Neuroinflammation. 2015;12:61.

13. Sun H, Gong S, Carmody RJ, Hilliard A, Li L, et al. TIPE2, a negative regulator of innate and adaptive immunity that maintains immune homeostasis. Cell. 2008;133:415-26.

14. Zhang Y, Shao Z, Zhang X, Jia X, Xia Y, et al. TIPE2 Play a Negative Role in TLR4-Mediated Autoimmune $T$ Helper 17 Cell Responses in Patients with Myasthenia Gravis. J Neuroimmune Pharmacol. 2015;10:635-44.

15. Peng Y, Zhao Q, Zhang H, Han B, Liu S, et al. TIPE2, a negative regulator of TLR signaling, regulates p27 through IRF4-induced signaling. Oncol Rep. 2016;35:2480-6. 
16. Ding J, Su J, Zhang L, Ma J. Crocetin Activates Foxp3 Through TIPE2 in Asthma-Associated Treg Cells. Cell Physiol Biochem. 2015;37:2425-33.

17. Qin B, Wei T, Wang L, Ma N, Tang Q, et al. Decreased expression of TIPE2 contributes to the hyperreactivity of monocyte to Toll-like receptor ligands in primary biliary cirrhosis. J Gastroenterol Hepatol. 2016;31:1177-83.

18. Li X. TIPE2 regulates tumor-associated macrophages in skin squamous cell carcinoma. Tumour Biol. 2016;37:5585-90.

19. Li P, Jiwu C. Butin Attenuates Brain Edema in a Rat Model of Intracerebral Hemorrhage by Anti Inflammatory Pathway. TransI Neurosci. 2018;9:7-12.

20. Sandgaard E, Troldborg A, Lauridsen SV, Gyldenholm T, Thiel S, et al. Changes in the Lectin Pathway Following Intracerebral or Spontaneous Subarachnoid Hemorrhage. Mol Neurobiol. 2019;56:78-87.

21. Zhao X, Ting SM, Sun G, Roy-O'Reilly M, Mobley AS, et al. Beneficial Role of Neutrophils Through Function of Lactoferrin After Intracerebral Hemorrhage. Stroke. 2018;49:1241-7.

22. MacLellan CL, Colbourne F. Mild to moderate hyperthermia does not worsen outcome after severe intracerebral hemorrhage in rats. J Cereb Blood Flow Metab. 2005;25:1020-9.

23. Power $C$, Henry S, Del Bigio MR, Larsen $\mathrm{PH}$, Corbett $\mathrm{D}$, et al. Intracerebral hemorrhage induces macrophage activation and matrix metalloproteinases. Ann Neurol. 2003;53:731-42.

24. Mayne M, Fotheringham J, Yan HJ, Power C, Del Bigio MR, et al. Adenosine A2A receptor activation reduces proinflammatory events and decreases cell death following intracerebral hemorrhage. Ann Neurol. 2001;49:727-35.

25. Fayngerts SA, Wang Z, Zamani A, Sun H, Boggs AE, et al. Direction of leukocyte polarization and migration by the phosphoinositide-transfer protein TIPE2. Nat Immunol. 2017;18:1353-60.

26. Ma Y, Liu X, Wei Z, Wang X, Wang Z, et al. The expression and significance of TIPE2 in peripheral blood mononuclear cells from asthmatic children. Scand J Immunol. 2013;78:523-8.

27. Lou Y, Liu S, Zhang C, Zhang G, Li J, et al. Enhanced atherosclerosis in TIPE2-deficient mice is associated with increased macrophage responses to oxidized low-density lipoprotein. J Immunol. 2013;191:4849-57.

28. Kong L, Liu K, Zhang YZ, Jin M, Wu BR, et al. Downregulation of TIPE2 mRNA expression in peripheral blood mononuclear cells from patients with chronic hepatitis C. Hepatol Int. 2013;7:8449.

29. Wang Z, Fayngerts S, Wang P, Sun H, Johnson DS, et al. TIPE2 protein serves as a negative regulator of phagocytosis and oxidative burst during infection. Proc Natl Acad Sci U S A. 2012;109:15413-8.

30. Lou Y, Sun H, Morrissey S, Porturas T, Liu S, et al. Critical roles of TIPE2 protein in murine experimental colitis. J Immunol. 2014;193:1064-70.

31. Li XM, Su JR, Yan SP, Cheng ZL, Yang TT, et al. A novel inflammatory regulator TIPE2 inhibits TLR4mediated development of colon cancer via caspase-8. Cancer Biomark. 2014;14:233-40. 
32. Lou Y, Zhang G, Geng M, Zhang W, Cui J, et al. TIPE2 negatively regulates inflammation by switching arginine metabolism from nitric oxide synthase to arginase. PLoS One. 2014;9:e96508.

33. Jiang Y, Li Q, Zhang Y, Gao Y, Jiang L, et al. TIPE2 governs macrophage polarization via negative regulation of mTORC1. Mol Med Rep. 2018;17:952-60.

34. Li F, Zhu X, Yang Y, Huang L, Xu J. TIPE2 Alleviates Systemic Lupus Erythematosus Through Regulating Macrophage Polarization. Cell Physiol Biochem. 2016;38:330-9.

35. Yuan B, Fu F, Huang S, Lin C, Yang G, et al. C5a/C5aR pathway plays a vital role in brain inflammatory injury via initiating Fgl-2 in intracerebral hemorrhage. Mol Neurobiol. 2017;54:618797.

36. Zhang Y, Mei S, Zhou Y, Yang D, Pan T, et al. TIPE2 negatively regulates mycoplasma pneumoniatriggered immune response via MAPK signaling pathway. Sci Rep. 2017;7:13319:1-8.

\section{Figures}
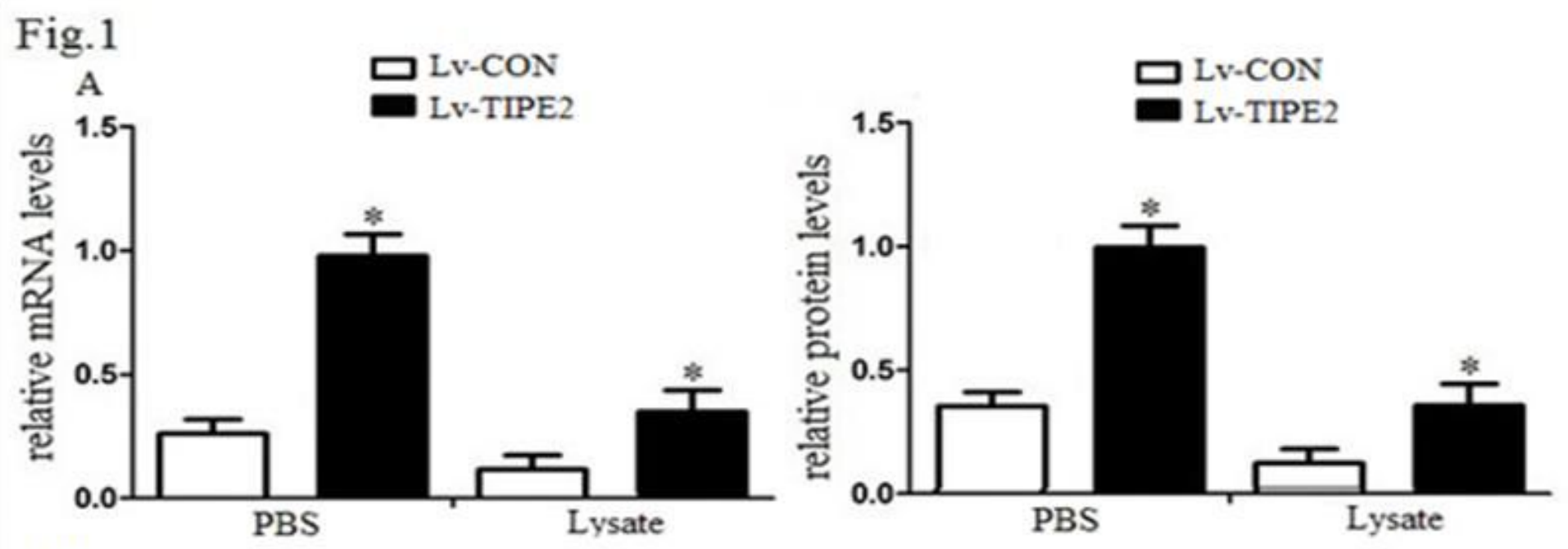

\section{B}

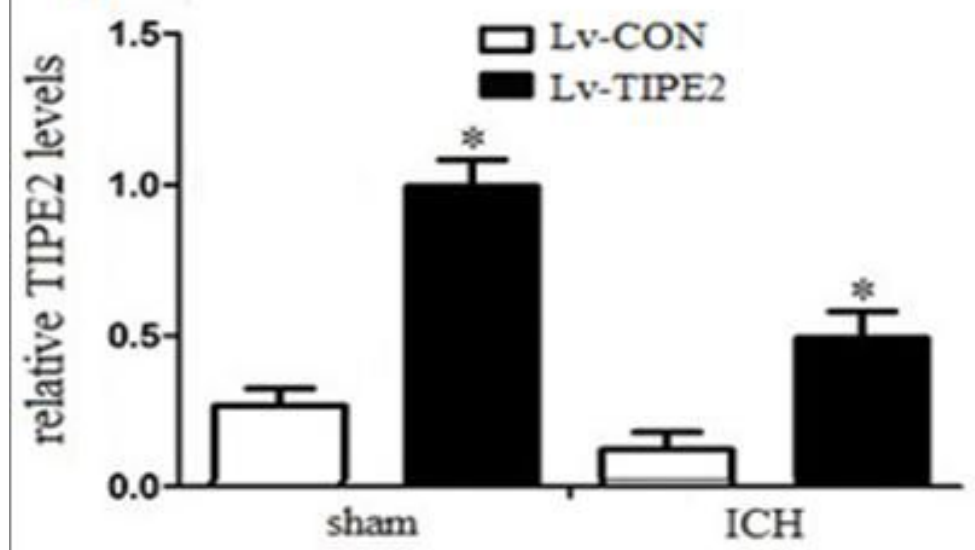

Figure 1

TIPE2 lentivirus improved TIPE2 levels following erythrocyte lysate treatment and ICH. (A) TIPE2 lentivirus transduced or control macrophages $(1 \times 105)$ were stimulated with $10 \mu \mathrm{L}$ of erythrocyte lysate or 
PBS. After 3 days, the supernatants were removed, and macrophages were collected for mRNA and protein analysis by RT-qPCR and western blotting. (D) The mice ( $n=10$ each group) were equally divided into the sham group and ICH group. After $3 \mathrm{ds}$, TIPE2 protein levels in the hippocampi of the mice were examined by western blot analysis. Experiments performed in triplicate showed consistent results. The data are presented as the mean \pm standard error of the mean (SEM) of three independent experiments. ${ }^{*} \mathrm{P}$ $<0.05$.

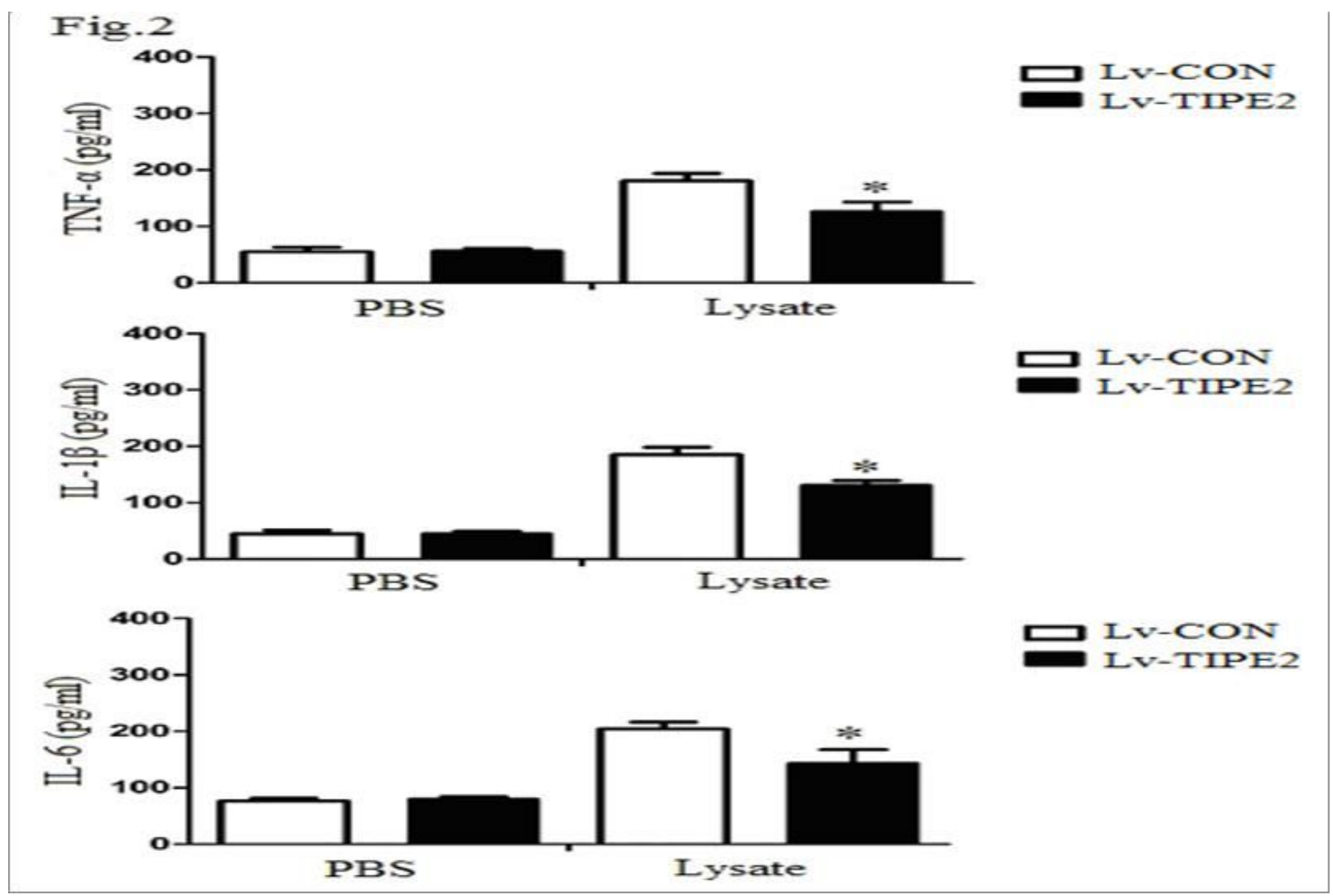

Figure 2

TIPE2 lentivirus decreased the macrophage inflammatory response in vitro. TIPE2 lentivirus transduced or control macrophages $(1 \times 105)$ were stimulated with $10 \mu \mathrm{L}$ of erythrocyte lysate or PBS. After 3 days, the supernatants were removed, and TNF- $a$, IL- $1 \beta$ and IL- 6 cytokine production was further analyzed by ELISA. Experiments performed in triplicate showed consistent results. The data are presented as the mean \pm SD of three independent experiments. ${ }^{*} \mathrm{P}<0.05$. 
Fi으.3

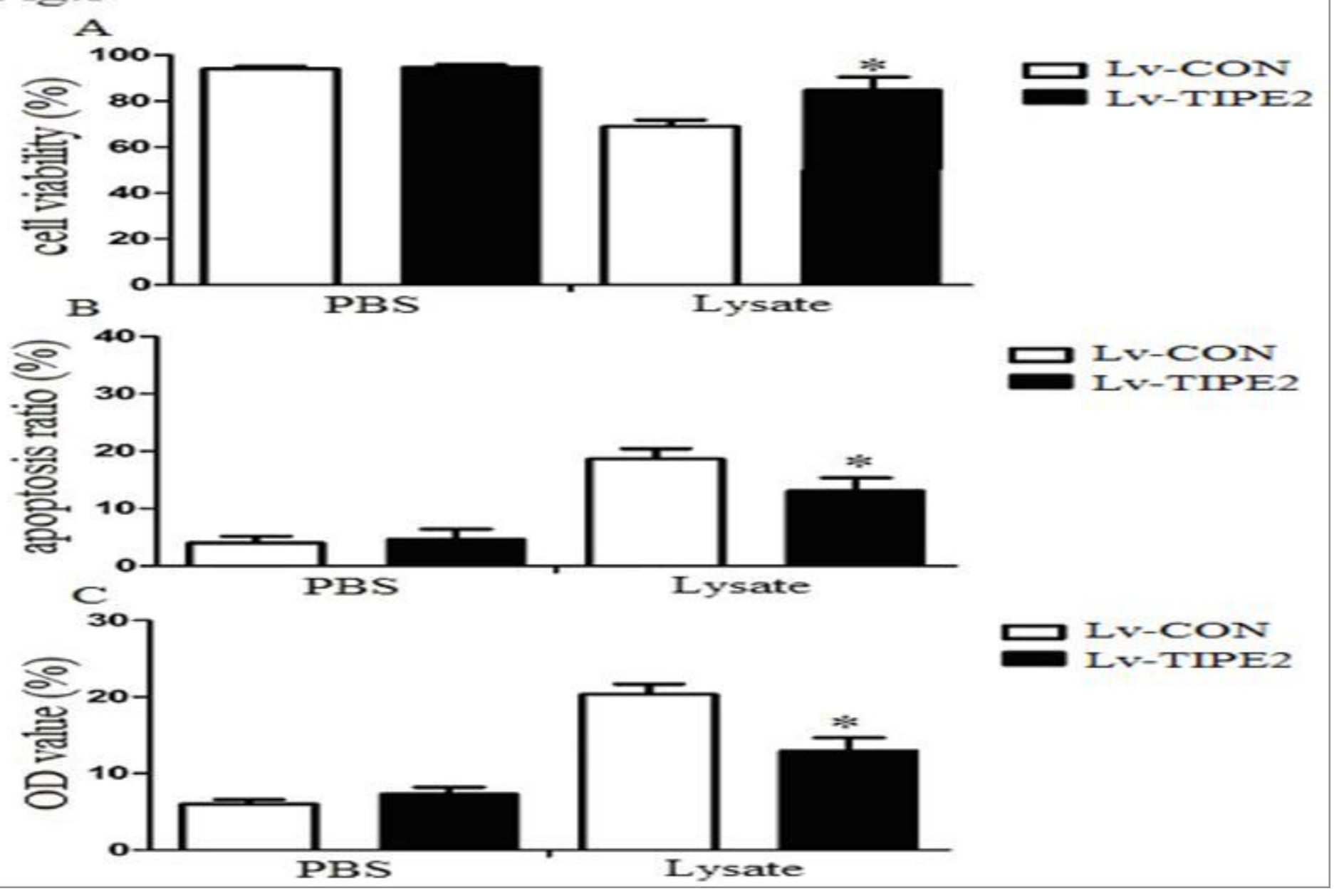

Figure 3

TIPE2 lentivirus inhibited macrophage-mediated inflammatory injury in neurons (A) Neurons were treated with conditioned medium from erythrocyte lysate-treated TIPE2 lentivirus transduced or control macrophages. After 48 h, MTT reagent was added, and cell viability was assessed. (B) After 48 h, neurons were treated with conditioned medium from erythrocyte lysate-treated TIPE2 lentivirus transduced or control macrophages, and the cell apoptosis ratio was detected by flow cytometry. Apoptotic cells were identified as annexin V-positive and propidium iodide (PI)-negative cells. (C) After 48 $h$, neurons were treated with conditioned medium from erythrocyte lysate-treated TIPE2 lentivirus transduced or control macrophages, and the ApoAlertcaspase-3 Colorimetric Assay Kit (Clontech, Palo Alto, USA) was used. Hydrolyzed pNA was detected using a Multiscan MS colorimeter (Thermo Labsystems, Vantaa, Finland) at $405 \mathrm{~nm}$. Experiments performed in triplicate showed consistent results. The data are presented as the mean \pm SD of three independent experiments. ${ }^{*} \mathrm{P}<0.05$. 
Fig.4

Lv-CON (sham)

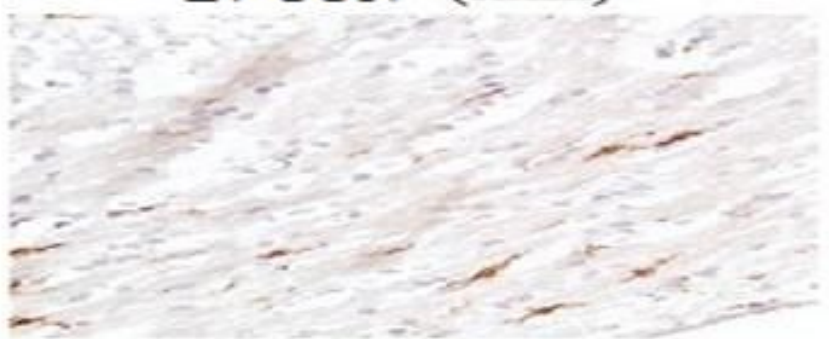

LV-TIPE2 (sham)

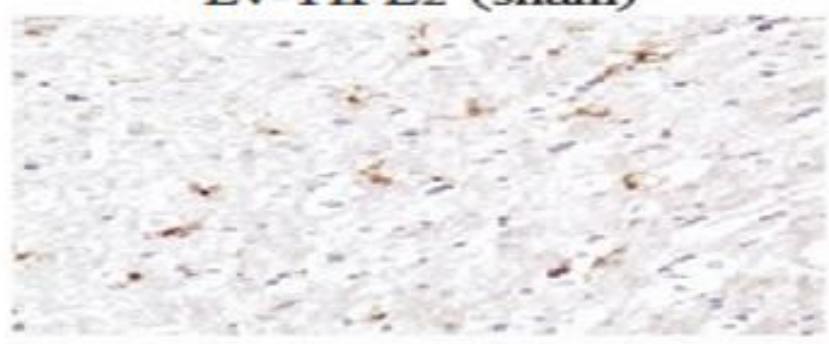

Lv-CON $(\mathrm{ICH}$

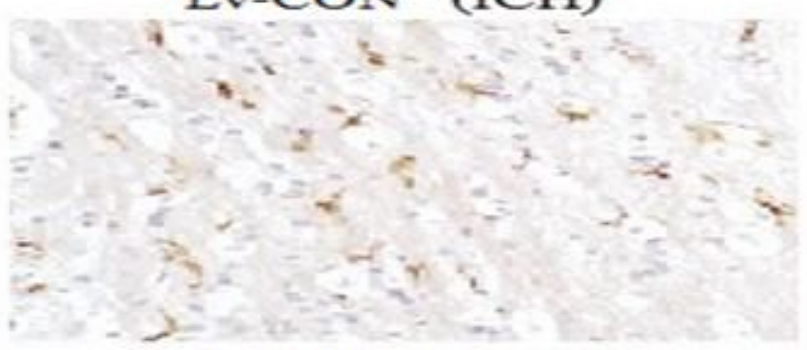

LV-TIPE2 ( $\mathrm{CH})$

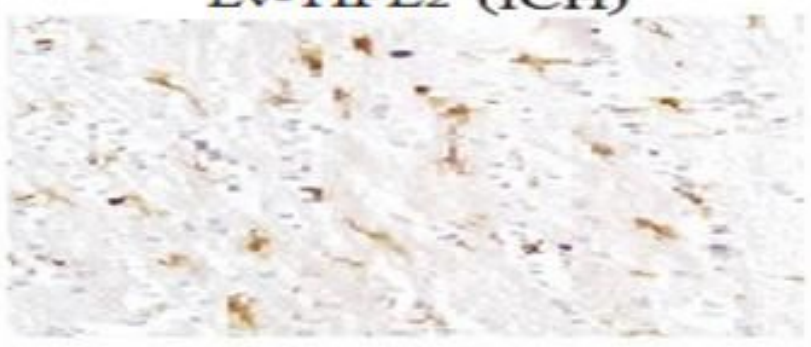

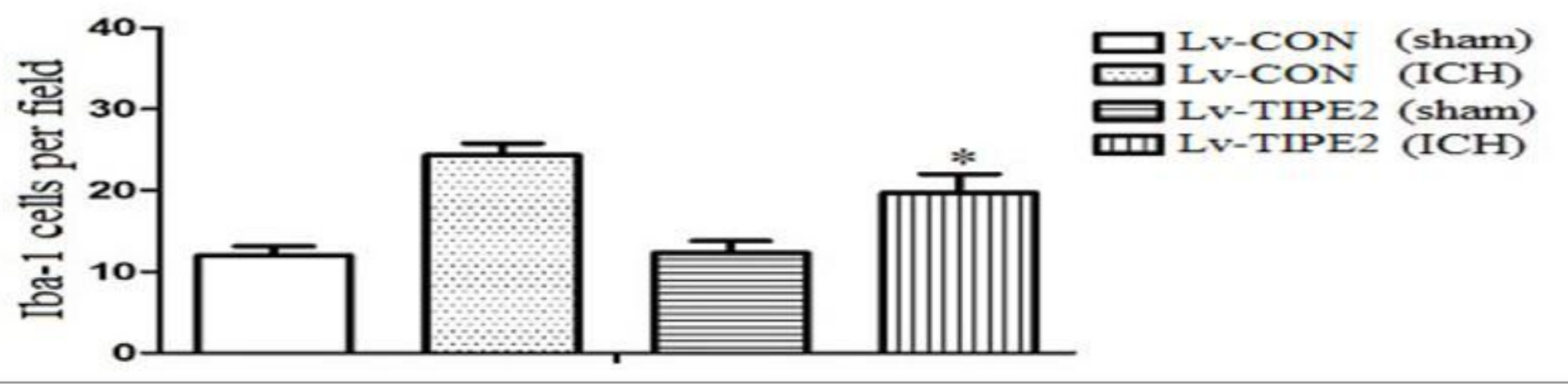

\section{Figure 4}

TIPE2 lentivirus attenuated macrophage accumulation in the perihematomal region Three days after ICH, mice ( $n=10$ per group) were deeply anesthetized transcardially. The brains were removed and postfixed. The perihematomal region of cerebral tissue was collected, and macrophages were analyzed with an antiIba-1 antibody ( $\times 400$ magnification). Experiments performed in triplicate showed consistent results. The data are presented as the mean \pm standard error of mean (SEM) of three independent experiments. *P < 0.05 . 


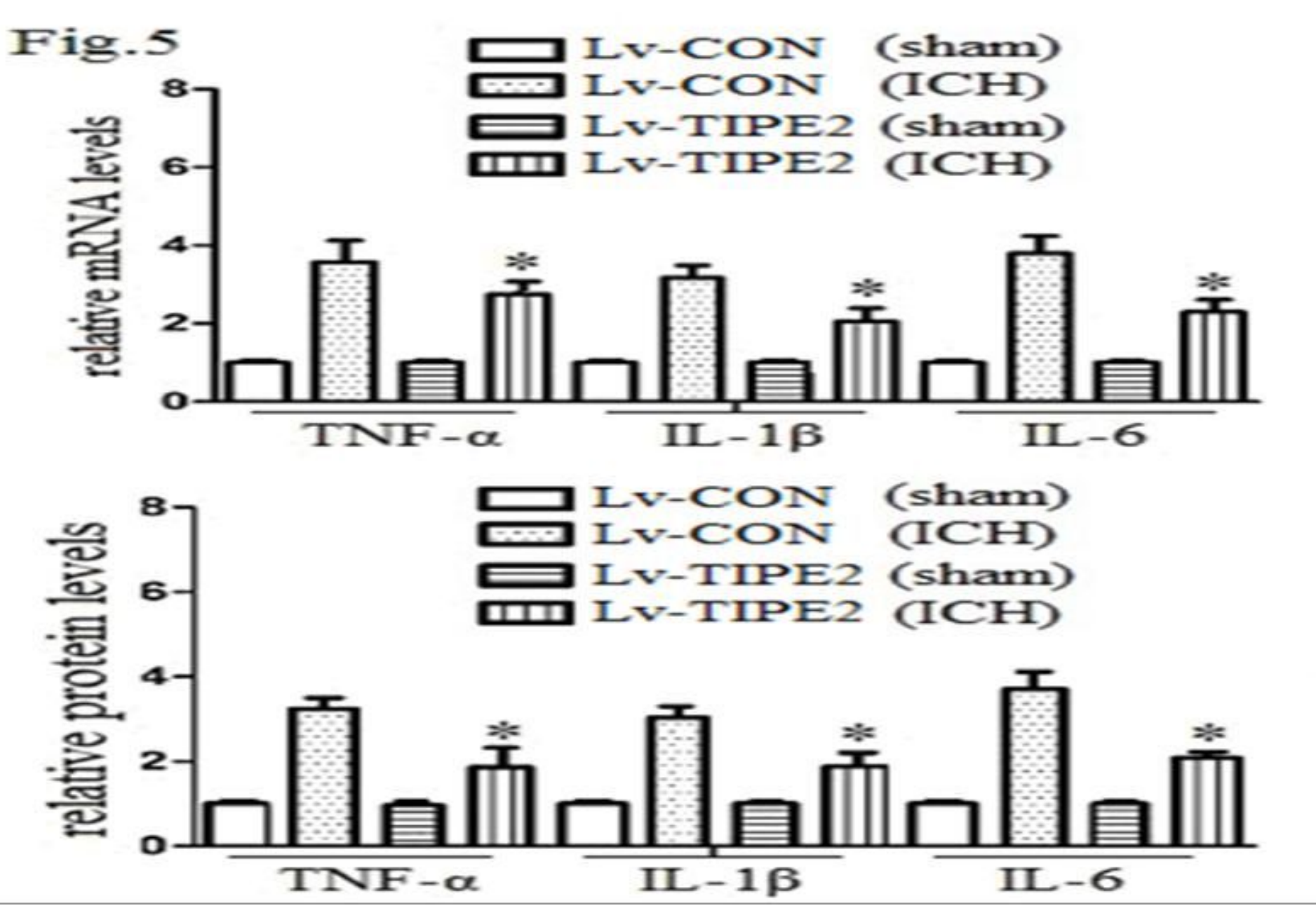

Figure 5

TIPE2 lentivirus inhibited inflammation in the perihematomal region. Three days after $\mathrm{ICH}$, mice $(\mathrm{n}=10$ per group) were deeply anesthetized transcardially. The brains were removed and postfixed. The perihematomal region of cerebral tissue was collected, and the tissue lysates were further analyzed for cytokine production by qRT-PCR and western blotting. Experiments performed in triplicate showed consistent results. The data are presented as the mean \pm standard error of mean (SEM) of three independent experiments. ${ }^{*} \mathrm{P}<0.05$. 
Fig.6A

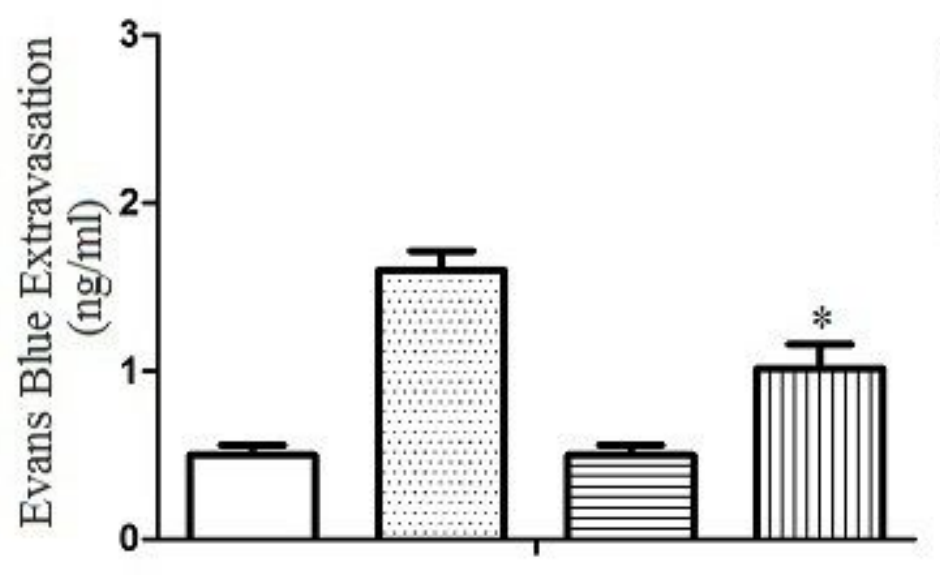

Fig.6B
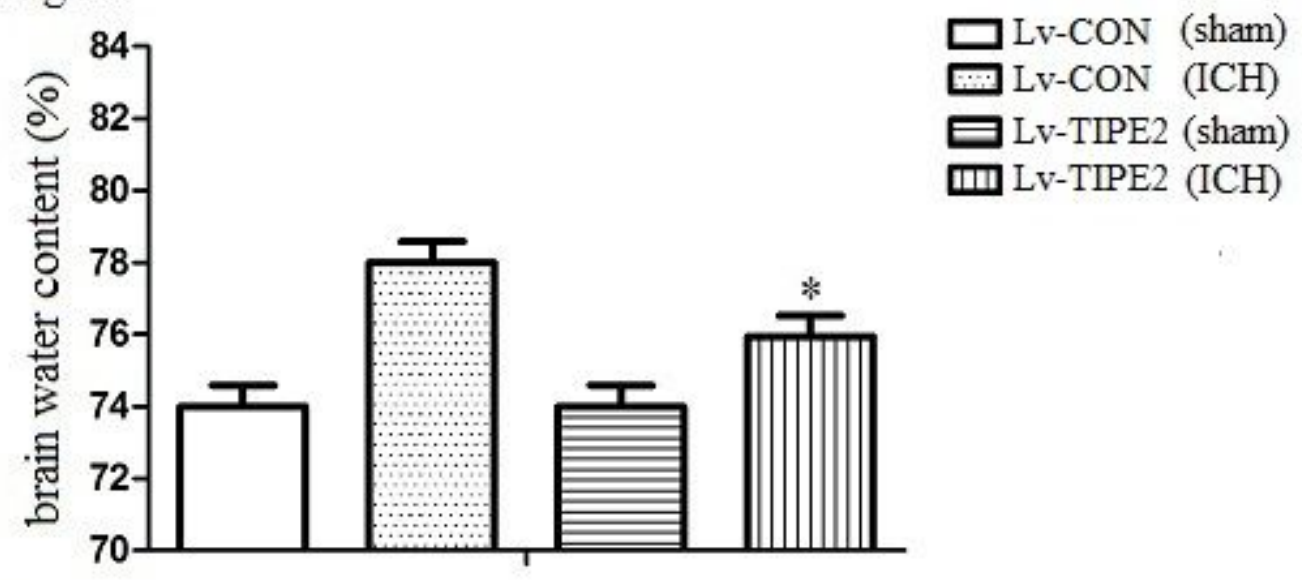

Fig.6C

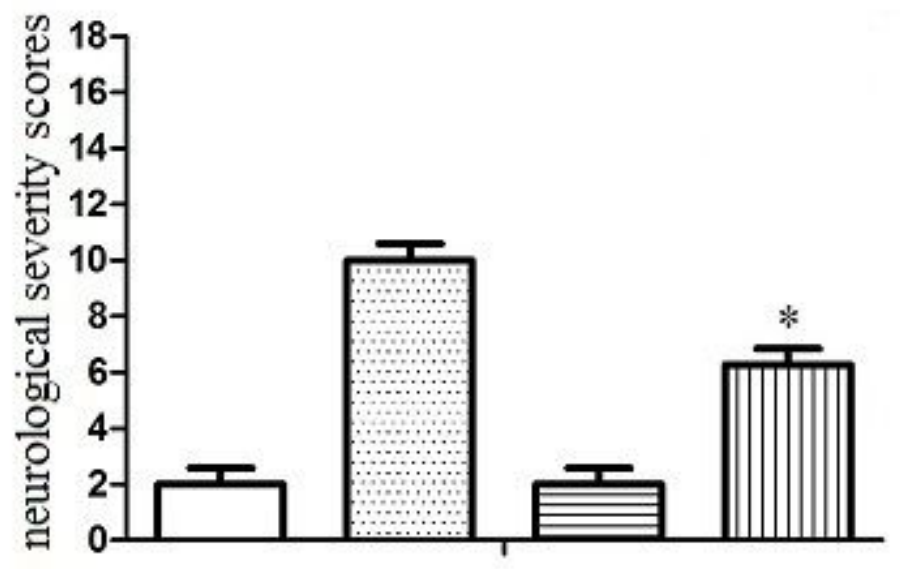

Figure 6

TIPE2 lentivirus attenuated neurological damage following ICH. (A) Three days after ICH, BBB disruption in the mice ( $n=10$ per group) was analyzed. (B) Three days after $\mathrm{ICH}$, the cerebral water content of mice ( $n=10$ per group) was also analyzed. (C) Three days after $\mathrm{ICH}$, the mice $(\mathrm{n}=10$ per group) were in stable condition, and neurological deficit tests were performed by measuring behaviors, including a composite of motor, sensory, reflex, and balance behaviors. Experiments performed in triplicate showed consistent 
results. The data are presented as the mean \pm standard error of mean (SEM) of three independent experiments. ${ }^{*} \mathrm{P}<0.05$. 